\title{
Analysis of phosphorylation status of ectopically expressed proteins in early Xenopus embryos
}

\section{Short title:}

Phospho-status of ectopically expressed protein

\section{Authors:}

Laura J.A. Hardwick ${ }^{1,3}$ and Anna Philpott ${ }^{1,2}$

\author{
Affiliations: \\ ${ }^{1}$ Hutchison/MRC Research Centre, Department of Oncology, University of Cambridge, \\ Cambridge, CB2 0XZ, UK. \\ ${ }^{2}$ Wellcome-MRC Cambridge Stem Cell Institute, University of Cambridge, Cambridge, UK. \\ ${ }^{3}$ Peterhouse, University of Cambridge, Cambridge, CB2 1RD, UK.
}

\section{Corresponding author:}

Professor Anna Philpott: (+44) 1223 762675; ap113@cam.ac.uk

\begin{abstract}
:
Xenopus embryos provide a rapid and accessible in vivo model, expressing a plethora of endogenous kinase and phosphatase enzymes that control protein phosphorylation, often affecting physiological function. Traditionally, detection of protein phosphorylation has been achieved by radioisotope phosphate labelling of proteins, sometimes with in vitro assays using recombinant proteins, or by site-specific phospho-antibodies. However, target phospho-sites and kinases responsible are often unknown, and use of radioactive isotopes is not always desirable. Therefore, as a first step to determining functional significance of potential phosphorylation, it is useful to show that a protein can be phosphorylated in vivo in Xenopus eggs and/or embryos. This protocol describes a non-radioactive method to visualise protein phosphorylation by exposing the protein to the egg/embryo kinase environment and observing a difference in protein migration on SDS-PAGE and western blot with and without treatment with lamda phosphatase enzyme. Subsequent investigation of the ability of site-
\end{abstract}


specific phospho-mutant proteins to recapitulate the effect of phosphatase treatment can be used to explore the identity of the phosphorylated sites. Moreover, detection of multiple bands of the protein of interest even after phosphatase treatment points to the presence of other types of post-translational modifications.

\section{Materials:}

It is essential that you consult the appropriate Material Safety Data Sheets and your institution's Health and Safety Office for proper handling of equipment and hazardous material used in this protocol.

RECIPES: Please see the end of this protocol for recipes indicated by $\langle\mathrm{R}\rangle$. Additional recipes can be found online at http://cshprotocols.cshlp.org.site/recipes

\section{Reagents:}

- Antibodies for western blot - antibody for protein of interest; antibody for loading control - e.g. anti- $\alpha$-tubulin.

- Embryo lysis buffer $\langle\mathrm{R}\rangle$. Prepare fresh and hold on ice.

- Fertilisation and embryo culture reagents; mRNA encoding proteins of interest, - see Protocol: Microinjection of mRNAs and Oligonucleotides (Moody 2018).

- Lamba protein phosphatase kit with enzyme and associated 10x PMP Buffer and $10 \mathrm{mM} \mathrm{MnCl} 2$ (e.g. NEB).

- Protein quantification assay - e.g. BCA assay.

- Sample buffer with reducing agent for SDS-PAGE.

- Western blotting reagents - see Protocol: A rapid western blotting method for the Xenopus oocyte (Lin-Moshier and Marchant 2013). The gel percentage and composition required for optimal separation of phospho-forms by SDS-PAGE will depend on the protein of interest.

Equipment:

- Centrifuge - benchtop, temperature controlled and chilled to $4^{\circ} \mathrm{C}$.

- Dry ice <optional> Required if freezing embryos prior to protein extraction.

- Glass pipette with rubber suction - 1x wide bore and 1x narrow bore.

- Heat block at $70^{\circ} \mathrm{C}$.

- Ice bucket with wet ice. 
- Microcentrifuge tubes $-1.5 \mathrm{ml}$.

- Micro-injection equipment for Xenopus embryos - see (Moody 2018).

- Protein quantification equipment.

- Water bath at $30^{\circ} \mathrm{C}$.

- Western blotting equipment - see (Lin-Moshier and Marchant 2013)

Method:

This protocol describes a rapid and non-radioactive method to visualise potential in vivo phosphorylation of an over-expressed protein of interest in Xenopus embryos. This is achieved by comparing migration of embryo extracts after SDS-PAGE and western blotting with and without lambda protein phosphatase treatment, avoiding the need for phospho-site specific antibodies. This complements in vitro kinase assays with recombinant target protein and kinase enzymes, and has the added benefit of utilising the endogenous kinase and phosphatase enzymes in the embryo, to allow determination of phosphorylation in vivo at the developmentally relevant stage. This method can also be used to demonstrate the importance of specific phosphorylation sites by comparing migration of wild-type and mutant versions of the protein of interest, where individual or a combination of potential phosphorylation sites have been mutated to a non-phosphorylatable amino acid such as Alanine [e.g. (Hardwick and Philpott 2015; Hardwick et al. 2016)]. The protocol can be conducted in continuous or multiple discrete steps as described below.

Preparation of embryos

1. Microinject mRNA encoding each protein of interest into a separate dish of one or two cell-stage Xenopus embryos, keeping one dish as uninjected control embryos. See (Moody 2018).

The amount of mRNA to be injected will require individual optimisation for the protein of interest.

2. Culture embryos to the relevant stage for analysis of the protein of interest.

3. Carefully transfer $10-15$ embryos to a clean $1.5 \mathrm{ml}$ microcentrifuge tube using a wide-bore glass pipette.

The number of embryos collected can be altered if larger volumes of protein extract are required. 
4. Remove as much of the culture media as possible using a narrow bore glass pipette, and either proceed directly to protein extraction stages or snap freeze embryos in dry ice and store at $-80^{\circ} \mathrm{C}$.

Additional buffer may be removed with a $10 \mu$ l pipette but take care not to lyse embryos prematurely.

Protein extraction and quantification from whole embryos

This part of the procedure will take approximately 1 hour and should be conducted on ice. The protein extracts can be used directly or stored at $-20^{\circ} \mathrm{C}$.

3. If embryos have previously been frozen, thaw on ice while making up fresh embryo lysis buffer and pre-chilling the centrifuge to $4^{\circ} \mathrm{C}$.

4. Homogenise embryos by repeated pipetting in embryo lysis buffer, using $4 \mu 1-10 \mu l$ per embryo.

$4 \mu l$ is suggested per embryo for unstable proteins or proteins expressed at a low level to increase total protein concentration in the extract.

5. Spin at $4{ }^{\circ} \mathrm{C}$ for 6 minutes at $1200 \mathrm{xg}$ and collect the middle fraction, above the pellet and below the lipid layer.

6. Re-spin the collected fraction at $4^{\circ} \mathrm{C}$ for 6 minutes at $1200 \mathrm{xg}$ and collect the supernatant as the protein extract.

7. Determine total protein concentration in each extract using a $1 \mu 1$ aliquot - e.g. by BCA assay, using manufacturer's protocol.

8. <Optional> Conduct SDS-PAGE and western blot with whole embryo protein extract to optimise loading amounts required for adequate visualisation and to determine the gel conditions for optimal protein separation.

The total protein amounts required for detection will vary depending on the protein of interest and may range from $20-80 \mu \mathrm{g}$. If protein concentration needs to be increased to enable sufficient total protein in the loading volume, embryos can be lysed in a reduced volume in step 4.

Lambda protein phosphatase treatment

This part of the procedure will take approximately 1.5 hours but can be done in isolation using pre-frozen protein extract and prior to SDS-PAGE. The protein phosphatase enzyme is 
very sensitive to temperature so must be stored at $-80^{\circ} \mathrm{C}$ and only defrosted on ice for the minimum time possible.

9. Select the amount of total protein from each extract to be used for SDS-PAGE and western blot - e.g. by optimisation in step 8 .

10. Set up protein phosphatase reactions (and control reactions without enzyme) on ice, according to manufacturer's instructions, leaving the phosphatase enzyme at $-80^{\circ} \mathrm{C}$ until immediately prior to use and then snap freeze on dry ice as soon as possible.

Lamba protein phosphatase is a $\mathrm{Mn}^{2+}$-dependent protein phosphatase with activity towards phosphorylated serine, threonine and tyrosine residues.

Each protein of interest should have duplicate reactions in a total volume of $20 \mu \mathrm{l}$;

phosphatase enzyme is added to one reaction and an equivalent volume of water is added to the other.

Due to glycerol content in the enzyme preparation, keep the concentration of enzyme to less than $10 \%$.

11. Incubate all reactions (with and without phosphatase) at $30^{\circ} \mathrm{C}$ in a water bath for 1 hour. 12. Add the required amount of SDS-reducing loading buffer and heat at $70^{\circ} \mathrm{C}$ for 10 minutes on a heat block.

These samples can be run directly on SDS-PAGE or frozen at $-80^{\circ} \mathrm{C}$. If samples are stored, repeat heat treatment of $70^{\circ} \mathrm{C}$ for 10 minutes prior to gel loading.

\section{Analysis by SDS-PAGE and western blotting}

13. Separate proteins by SDS-PAGE and western blotting. A loading control such as alphatubulin can be used to demonstrate equal loading.

The pair of samples for each protein of interest (with and without phosphatase treatment) should be run side-by-side to enable direct comparison of migration. An equivalent amount of total protein from uninjected embryos can be used to demonstrate antibody specificity for the over-expressed protein.

14. Compare protein migration in the sample lanes to determine the effect of phosphatase treatment.

Wild-type proteins may be phosphorylated on one or more sites, resulting in the appearance of a broad band or multiple discrete bands on western blot. The removal of these phosphate 
groups by phosphatase enzyme results in the appearance of a narrower, faster migrating band. Example images to illustrate these results are shown in (Hardwick and Philpott 2015; Hardwick et al. 2016). Discrete bands may still be present if the protein undergoes alternative post-translational modification such as ubiquitination or sumoylation.

Different SDS-PAGE conditions may be required for optimal separation of protein phosphoforms and demonstration of phospho-shift with phosphatase treatment. A higher percentage gel such as an $18 \%$ gel can aid separation. Alternatively, Phos-tag gels may provide superior resolution of discrete phospho-forms - see (Horinouchi et al. 2016). If phos-tag gels are used, an equivalent amount of $\mathrm{MnCl}_{2}$ must be added to the samples prior to phosphatase treatment to bind the EDTA in the embryo lysis buffer. This EDTA will otherwise chelate the $\mathrm{Mn}^{2+}$ of the gel and prevent detection of the phospho-forms.

\section{Acknowledgements:}

We thank Daniel Marcos Corchado for advice on Phos-tag SDS-PAGE.

\section{References:}

Hardwick LJ, Davies JD, Philpott A. 2016. MyoD phosphorylation on multiple C terminal sites regulates myogenic conversion activity. Biochemical and biophysical research communications 481: 97-103.

Hardwick LJ, Philpott A. 2015. Multi-site phosphorylation regulates NeuroD4 activity during primary neurogenesis: a conserved mechanism amongst proneural proteins. Neural development 10: 15.

Horinouchi T, Terada K, Higashi T, Miwa S. 2016. Using Phos-Tag in Western Blotting Analysis to Evaluate Protein Phosphorylation. Methods in molecular biology 1397: 267-277.

Lin-Moshier Y, Marchant JS. 2013. A rapid Western blotting protocol for the Xenopus oocyte. Cold Spring Harbor protocols 2013.

Moody SA. 2018. Microinjection of mRNAs and Oligonucleotides. Cold Spring Harbor protocols.

\section{Recipes:}

Embryo lysis buffer

Prepare fresh and store on ice during the protocol. $\beta$-glycerophosphate is included as a phosphatase inhibitor but additional inhibitors or competitors may be required depending on the stability of the phosphorylation status of the protein of interest. 


\begin{tabular}{|l|l|}
\hline Reagent & $\begin{array}{l}\text { Final concentration } \\
(\mathbf{1 X})\end{array}$ \\
\hline Tris pH8.0 & $50 \mathrm{mM}$ \\
\hline $\mathrm{NaCl}$ & $100 \mathrm{mM}$ \\
\hline EDTA pH8.0 & $5 \mathrm{mM}$ \\
\hline TritonX-100 & $0.10 \%$ \\
\hline$\beta$-glycerophosphate & $50 \mathrm{mM}$ \\
\hline $\begin{array}{l}\text { Protease Inhibitor } \\
\text { Cocktail (PIC); } \\
\text { Roche }\end{array}$ & $1 \mathrm{x}$ \\
\hline
\end{tabular}

